\title{
Derivation of 30 human embryonic stem cell lines-improving the quality
}

\author{
Susanne Ström • Frida Holm • Rosita Bergström • \\ Anne-Marie Strömberg • Outi Hovatta
}

Received: 1 April 2009 /Accepted: 23 January 2010 /Published online: 3 March 2010 / Editor: P. Andrews

(C) The Author(s) 2010. This article is published with open access at Springerlink.com

\begin{abstract}
We have derived 30 human embryonic stem cell lines from supernumerary blastocysts in our laboratory. During the derivation process, we have studied new and safe method to establish good quality lines. All our human embryonic stem cell lines have been derived using human foreskin fibroblasts as feeder cells. The 26 more recent lines were derived in a medium containing serum replacement instead of fetal calf serum. Mechanical isolation of the inner cell mass using flexible metal needles was used in deriving the 10 latest lines. The lines are karyotypically normal, but culture adaptation in two lines has been observed. Our human embryonic stem cell lines are banked, and they are available for researchers.
\end{abstract}

Keywords Human embryonic stem cells · Feeder cells . Human fibroblasts · Defined medium

\section{Introduction}

Human embryonic stem cells (hESC), first reported in 1984 (Fishel et al. 1984), and as permanent lines $10 \mathrm{yr}$ ago (Thomson et al. 1998; Reubinoff et al. 2000), are for the time being in the center of biological and medical research. They have great potential for use in clinical applications in regenerative medicine due to their ability to form all the tissue types in the body. Before clinical applications, many

Susanne Ström and Frida Holm contributed equally to this work.

S. Ström • F. Holm • R. Bergström $(\triangle) \cdot$ A.-M. Strömberg $•$

O. Hovatta

Department of Clinical Science, Technology and Intervention,

Karolinska Institutet, Karolinska University Hospital Huddinge,

SE 14186 Stockholm, Sweden

e-mail: rosita.bergstrom@ki.se quality and safety requirements have to be met (Crook et al. 2007). In our derivations, we have developed culture systems in which we could at first avoid mouse fibroblasts as feeder cells and then fetal calf serum (FCS) and immunosurgery, which all involve animal proteins which are immunogenic (Martin et al. 2005) and bear a risk of microbial contamination (Unger et al. 2008). We have derived in our laboratory $30 \mathrm{hESC}$ lines. They were derived from blastocyst stage supernumerary embryos donated for this purpose by couples treated in the in vitro fertilization (IVF) unit of our hospital.

We have successfully derived and cultured them on postnatal human foreskin fibroblasts (hff), completely avoiding the use of animal cells as feeders (Hovatta et al. 2003). Our second step toward clinical grade hESC lines was to use a serum replacement (SR) as a constituent of the medium, first in culture of the existing lines, instead of FCS (Koivisto et al. 2004) and then also in the derivation medium of the following lines (Inzunza et al. 2005).

To further minimize the use of animal-derived substances, we developed a method to mechanically isolate the inner cell mass (ICM) to replace the use of immunosurgery (Strom et al. 2007) that involves animal components and enzymes. For the time being, we have derived $10 \mathrm{hESC}$ lines using mechanical isolation of the ICM.

Here, we describe the derivation procedures of our 30 hESC lines.

\section{Materials and Methods}

Legal and ethics framework in Sweden and embryos used. The Swedish law allows derivation and culture of hESC lines from human supernumerary IVF embryos after both partners' informed consent. No reimbursement is allowed. 
According to a law which came into force in 2005, establishing embryos for research purposes has also been allowed in particular situations which cannot be solved by any other method, under the approval of a regional ethics board.

We have ethics approval from the Ethics Board of Karolinska Institutet and the Regional Ethics Board in Stockholm for derivation and culture, expansion, banking, and differentiation of hESC lines from supernumerary embryos. We also have an approval for studying the possibility for obtaining hESC lines using somatic cell nuclear transfer (SCNT) to in vitro matured oocytes which could not be injected by sperm in treatment of male infertility. We have only obtained a few oocytes for this study, and we have not managed to derive any hESC lines using SCNT. We are now focusing more to induced pluripotent cells in achieving patient-specific cell lines.

All our lines have been derived from blastocyst stage embryos which were donated by couples undergoing IVF treatment in our hospital. Only embryos which could not be used in the couples' infertility treatment were used for hESC derivation. During the treatment process, the best embryo is transferred to the woman, occasionally two if the embryo quality is scored as poor or the couple has undergone more than two unsuccessful treatment cycles earlier. The rest of the embryos which meet the score of at least 2 out of 3.5 (Fridstrom et al. 1999) are cryopreserved. The low scoring embryos were discarded or could be donated for research until 2008, but now, all are cultured to blastocyst stage. If they reach this stage, they will be vitrified for the couple's possible later infertility treatment. The maximum cryopreservation time for embryos in Sweden is $5 \mathrm{yr}$. If they are not used for infertility treatment before the end of this period, they are discarded or donated to research. During the first visit to the IVF unit, the couple is given both written and oral information regarding the research projects in the unit, including stem cell line derivation (Bjuresten and Hovatta 2003). The couples sign an informed consent form approved by the ethics committee. They do not get financial reimbursement for the donation. A specially educated midwife gave the counseling.

If there were, after the first embryo transfer, embryos which had too low score for cryopreservation at cleavage stage, or if the couple did not want to use the cryopreserved embryos for their treatment anymore, they could be donated for stem cell derivation. If the embryo was donated at cleavage stage to hESC derivation, the IVF laboratory cultured it to blastocyst stage before transfer to the stem cell laboratory.

In 2002-2009, we have used 236 blastocysts in stem cell derivation.

Embryo culture. The embryos were cultured in IVF medium (Vitrolife, Göteborg, Sweden) individually in $20-\mu$ l droplets under oil for the first $2 \mathrm{~d}$ and then transferred to blastocyst medium (Vitrolife). From 2006 up until today, the early embryos are cultured in ISM1 and moved to ISM2 (both from Medicult, Jyllinge, Denmark) on Day 3. Oocyte and embryo culture was performed in $20-\mu l$ drops of culture medium under paraffin oil (Ovoil, Vitrolife) in an atmosphere of $6 \% \mathrm{CO}_{2}$ in air at $37^{\circ} \mathrm{C}$.

Isolation of the inner cell mass by immunosurgery. The first 20 hESC lines HS181-HS402 (Table 1) were derived by immunosurgery described earlier by Solter and Knowles (1975). The zona pellucida was first removed using 0.5\% Pronase (Sigma-Aldrich Co., St Louis, MO). The trophectoderm cells are then removed by using rabbit antihuman whole serum and guinea pig complement serum (both from Sigma-Aldrich). The isolated ICM was then plated on human foreskin fibroblast feeder cells.

Mechanical isolation of the inner cell mass. The blastocyst is put in a small drop of stem cell culture medium, and the zona pellucida is opened up with specially made flexible metal needles $(0.125 \mathrm{~mm}$ in diameter; Strom et al. 2007). These needles have now become commercially available (Hunter Scientific, Essex, UK). The embryo was fixed at the edge of the drop and opened up with the needles, and the ICM was dissected out. The procedure took 20-40 s under a stereomicroscope (Nikon 1500). The ICM was allowed to grow on the human skin feeder cells for $12-15 \mathrm{~d}$ before the first transfer of the growing cells to a new feeder plate.

Feeder cells. Human foreskin fibroblasts as feeder cells (CRL-2429; ATCC, Mananas, VA) have been used in all our derivations. Before being used as substrate cells, they had been irradiated with $40 \mathrm{~Gy}$. The medium used in culture of these feeder cells was Iscovs medium (Gibco Invitrogen Corp., Paisley, UK) supplemented with $10 \%$ FCS (FCS; SDS, Sweden).

Human embryonic stem cell derivation and maintenance cultures. The culture medium used for derivation of the first four hESC lines (HS181, HS207, HS235, HS237) was Knockout D-MEM 13, supplemented with $2 \mathrm{nmol} / 1$ L-glutamine (both from Gibco), 20\% fetal bovine serum (FBS; R\&D, Sweden), $0.1 \mathrm{mmol} / 1 \quad b$-mercaptoethanol (Gibco), 1\% nonessential amino acids (Gibco), and recombinant human LIF, $1 \mu \mathrm{l} / \mathrm{ml}$ (Chemicon, Temecula, CA; Hovatta et al. 2003). The later derivations of hESC lines HS293-HS539 have been derived and cultured in Knockout Dulbecco's modified Eagle's medium supplemented with $20 \%$ Knockout serum replacement, 2 mM Glutamax, $0.5 \%$ penicillin-streptomycin, $1 \%$ nonessential amino acids, $0.5 \mathrm{mM}$ 2-mercaptoethanol (all from Gibco), and $8 \mathrm{ng} / \mathrm{ml}$ basic fibroblast growth factor (bFGF; R\&D 
Systems, Oxon, UK) at $37^{\circ} \mathrm{C}$ in $5 \% \mathrm{CO}_{2}$ as previously described (Inzunza et al. 2005; Strom et al. 2007). We also tested derivation in X-vivo 10 (Cambrex Corporation, East Rutherford, NJ), but no lines were achieved from the 20 blastocysts used in this series.

During derivation and early culture, the hESCs were mechanically passaged in 5-7-d intervals by the use of a surgical scalpel. The colonies were lifted from the feeder layer in smaller pieces and then transferred onto fresh feeder cells.

At later passages, also enzymatic passaging using dispase $(5 \mathrm{mg} / \mathrm{mL}$, StemCell Tech Inc., Vancover, BC, Canada), collagenase type IV, and more recently, human recombinant trypsin, TrypLE Select/Express (all from Gibco) has been used but are not used as routine. Culture form single cell suspension has been feasible using a Rhokinase inhibitor Y-27632, (Merck Chemicals Ltd., Nottingham, UK; Martin-Ibanez et al. 2008).

All hESC lines that we have derived have also been cultured and maintained on a similar feeder layer of human foreskin fibroblasts and the same SR-supplemented culture medium as has been described for derivation procedures.

Feeder-free cultures have been used for many experiments, particularly those aimed at gene expression studies. Several culture systems have been tested, but our regular feeder-free culture matrix has been Matrigel (BD Biosciences, Bedford, MA) together with mTeSR1 Medium (StemCell Tech. Inc.), which supports the growth of all the lines.

We have recently developed a new human recombinant laminin 511 matrix which supports the long-term nondifferentiated growth of hESC together with a defined xeno-free medium (Rodin et al. 2010).

Vitrification. When a new hESC line has been derived, it is important to freeze cells at an early stage as possible. By using the open-pulled straw vitrification method described for the first time by Reubinoff et al. (2001), it is possible to freeze small amounts of cells. In short, ministraws were heat-softened over a hot plate and manually pulled until reaching optimal diameter. After cooling to room temperature, the straws were gamma irradiated (40 Gy). The two vitrification media that were used, VS1 and VS2, were both made from the same base of holding medium (HM), which included D-MEM containing HEPES buffer supplemented with 20\% SR (Gibco). VS1 contains 10\% dimethylsulfoxide (DMSO) and 10\% ethylene glycol, and VS2 contains $20 \%$ of both DMSO and ethylene glycol (Sigma-Aldrich) as well as $0.5 \mathrm{~mol} / 1$ sucrose. Cell aggregates were incubated in VS1 for $1 \mathrm{~min}$, followed by $25 \mathrm{~s}$ of incubation in VS2. The cell clumps were then washed in droplets of VS2 and then placed within a droplet of 1-2 $\mu$ VS2 from where the colonies where loaded by capillary force into the narrow end of the straw. The straw was then immediately submerged into liquid nitrogen and then stored for a long term.
The thawing of vitrified straws was also performed as described by Reubinoff and coworkers (2001). Directly from liquid nitrogen, the straw was held in room temperature for $3 \mathrm{~s}$, and the narrow end of the straw was submerged into SR-medium supplemented with $0.2 \mathrm{M}$ sucrose. When the medium within the straw was liquefied and medium started to fill up the straw, the wide opening of the straw was sealed. The straw was emptied into one well of a four-well plate containing SR-medium supplemented with $0.2 \mathrm{M}$ sucrose and incubated for $1 \mathrm{~min}$ in $37^{\circ} \mathrm{C}$. The cell aggregates were moved to the next well containing SRmedium supplemented with $0.1 \mathrm{M}$ sucrose and incubated for further $5 \mathrm{~min}$ in $37^{\circ} \mathrm{C}$. The cell aggregates were then moved to the third well containing SR-medium and incubated for $5 \mathrm{~min}$ in $37^{\circ} \mathrm{C}$. This step was repeated twice. The cells were then plated in a fresh feeder plate.

Slow freezing. When hESCs need to be frozen in higher scale, the slow freezing method is widely used (Reubinoff et al. 2001). Undifferentiated hESC colonies ( 25) were cut out from feeder layer using a surgical scalpel. Colonies were added into cryovials, and the freezing medium containing hESC culture medium without bFGF supplemented with $10 \%$ DMSO (Sigma-Aldrich) were added. The vial were placed in a Nalgene Cryo $1^{\circ} \mathrm{C}$ Freezing Container "Mr. Frosty" (Nalgene Nunc International, Rochester, NY) containing isopropanol and immediately put into $-70^{\circ} \mathrm{C}$. The next day, cryo vials were removed from $-70^{\circ} \mathrm{C}$ and placed into a liquid nitrogen tank.

Freezing with Rho-kinase inhibitor (Y-27632). In early 2009 , there was a method describing cryopreservation of dissociated hESCs in the presence of a ROCK inhibitor (Merck Chemicals, Ltd.; Martin-Ibanez et al. 2008). Briefly, cells that had been grown as single cells in present of ROCK inhibitor were harvested by adding ROCK inhibitor $(10 \mu \mathrm{M}) 1 \mathrm{~h}$ before detaching the cells from feeder layer with TrypLE ${ }^{\mathrm{TM}}$ express (Gibco). The dissociated cells were transferred to freezing medium without bFGF, supplemented with $10 \%$ DMSO. Between 30,000 and 100,000 hESCs were loaded into a cryo vial and frozen at $-70^{\circ} \mathrm{C}$ using freezing container as mentioned earlier and then stored in liquid nitrogen.

The thawing procedure of hESCs frozen in the presence of ROCK is the same as in slow freezing with some modifications. When seeding out hESCs onto fresh feeder cells, the culture medium should contain ROCK inhibitor $(10 \mu \mathrm{M})$. When the medium were changed for the first time after thawing, there were no longer necessary to add ROCK inhibitor to the culture medium.

Karyotyping. Karyotyping of new cell lines were carried out using standard G-banding techniques. Samples of 
hESCs were treated with colcemid KaryoMAX $(0.1 \mu \mathrm{g} / \mathrm{ml}$; Gibco) for $5 \mathrm{~h}$. The colcemid-treated cells were removed into a centrifugation tube and centrifuged at $1,300 \mathrm{rpm}$, for $7 \mathrm{~min}$. In the meantime, cell cultures were treated with trypsin for $5 \mathrm{~min}$. Supernatant were removed after centrifugation, and the trypsin-treated cells were added in the tube and resuspended. Prewarmed $0.0375 \mathrm{M} \mathrm{KCl}$ hypotonic solution were added in the tube and then incubated for $10 \mathrm{~min}$. Following centrifugation, the cells were resuspended in fixative of methanol and acetic acid (3:1). Metaphase spreads were prepared on glass microscope slides and G-banded by brief exposure to trypsin and stained with 4:1 Gurr's/Leishmann's stain (Sigma-Aldrich). A minimum of 10 metaphases was analyzed.
Teratomas. The pluripotency of the new line together with earlier control lines were tested in vivo as previously described (Inzunza et al. 2005). In brief, exponentially growing hESC lines were harvested from the culture plate using mechanical splitting. Five colonies $\left(10^{3}\right.$ to $10^{4}$ hESCs) were washed twice in phosphate-buffered saline (PBS) and put in a $1.5-\mathrm{mL}$ collection tube and $80-\mu \mathrm{L}$ SRcontaining culture medium, subsequently implanted beneath the testicular capsule of a young (7-wk-old) severe combined immunodeficiency/beige male mouse (C.B.-17/ GbmsTac-scid-bgDF N7, M\&B, Ry, Denmark). Teratoma growth was determined by palpation every week, and the mice were killed (cervical dislocation) $8 \mathrm{wk}$ after implantation. The teratoma was fixed, and sections were stained with hematox-

Table 1. The table includes all hESC lines derived at Karolinska Institutet between 2002 and 2009

\begin{tabular}{|c|c|c|c|c|c|c|}
\hline hESC Line & Day of Derivation & Isolation of the ICM & Medium Supplement & Feeder Layer & Karyotype & Teratoma Formation \\
\hline HS181 & 6 & Immunosurgery & $20 \% \mathrm{FCS}$ & hff & $46, X X$ & Yes \\
\hline HS207 & 7 & Immunosurgery & $20 \% \mathrm{FCS}$ & hff & $46, X Y$ & Yes \\
\hline HS235 & 6 & Immunosurgery & $20 \%$ FCS & hff & $46, X X$ & Yes \\
\hline HS237 & 6 & Immunosurgery & $20 \% \mathrm{FCS}$ & hff & $46, X X$ & Yes \\
\hline HS293 & 6 & Immunosurgery & $20 \% \mathrm{SR}$ & hff & $46, X Y$ & Yes \\
\hline HS306 & 5 & Immunosurgery & $20 \% \mathrm{SR}$ & hff & $46, X X$ & Yes \\
\hline HS346 & 7 & Immunosurgery & $20 \% \mathrm{SR}$ & hff & $46, X X$ & Yes \\
\hline HS351 & 6 & Immunosurgery & $20 \% \mathrm{SR}$ & hff & $46, X X$ & No \\
\hline HS360 & 6 & Immunosurgery & $20 \% \mathrm{SR}$ & hff & $46, X Y$ & Yes \\
\hline HS361 & 7 & Immunosurgery & $20 \% \mathrm{SR}$ & hff & $46, X Y$ & Yes \\
\hline HS362 & 6 & Immunosurgery & $20 \% \mathrm{SR}$ & hff & $46, X Y$ & Yes \\
\hline HS363 & 6 & Immunosurgery & $20 \% \mathrm{SR}$ & hff & $46, X Y$ & Yes \\
\hline HS364 & 6 & Immunosurgery & $20 \% \mathrm{SR}$ & hff & $46, X Y$ & Yes \\
\hline HS366 & 6 & Immunosurgery & $20 \% \mathrm{SR}$ & hff & $46, X X$ & Yes \\
\hline HS368 & 7 & Immunosurgery & $20 \% \mathrm{SR}$ & hff & $46, X Y$ & Yes \\
\hline HS380 & 6 & Immunosurgery & $20 \% \mathrm{SR}$ & hff & $46, X Y$ & Yes \\
\hline HS382 & 6 & Immunosurgery & $20 \% \mathrm{SR}$ & hff & $46, X Y$ & Yes \\
\hline HS400 & 6 & Immunosurgery & $20 \% \mathrm{SR}$ & hff & $46, \mathrm{XX}$ & Yes \\
\hline HS401 & 6 & Immunosurgery & $20 \% \mathrm{SR}$ & hff & $46, X Y$ & Yes \\
\hline HS402 & 6 & Immunosurgery & $20 \% \mathrm{SR}$ & hff & - & Yes \\
\hline HS415 & 6 & Mechanical & $20 \% \mathrm{SR}$ & hff & $46, X X$ & Yes \\
\hline HS420 & 6 & Mechanical & $20 \% \mathrm{SR}$ & hff & $46, X Y$ & Yes \\
\hline HS422 & 6 & Mechanical & $20 \% \mathrm{SR}$ & hff & $46, X Y$ & Yes \\
\hline HS426 & 8 & Mechanical & $20 \% \mathrm{SR}$ & hff & $46, X Y$ & Yes \\
\hline HS429 & 6 & Mechanical & $20 \% \mathrm{SR}$ & hff & $46, X X$ & Yes \\
\hline HS475 & 7 & Mechanical & $20 \% \mathrm{SR}$ & hff & - & Yes \\
\hline HS480 & 6 & Mechanical & $20 \% \mathrm{SR}$ & hff & - & Yes \\
\hline HS481 & 6 & Mechanical & $20 \% \mathrm{SR}$ & hff & - & - \\
\hline HS491 & 6 & Mechanical & $20 \% \mathrm{SR}$ & hff & - & - \\
\hline HS539 & 5 & Mechanical & $20 \% \mathrm{SR}$ & hff & - & - \\
\hline
\end{tabular}

The first $20 \mathrm{hESC}$ lines were derived by immunosurgery and the latest 10 by mechanical isolation of the inner cell mass. In the four first derivations, the medium was supplemented with fetal calf serum (FCS); this was later replaced by adding serum replacement (SR) to the media. All lines have not yet been karyotyped or tested for in vivo differentiation capacity 
ylin and eosin. The presence of tissue components of all three embryonic germ cell layers was shown, as analyzed from the stained sections (Inzunza et al. 2005).

All animal experiments were performed at the infectionfree animal facility of Karolinska University Hospital in accordance with ethical committee approval.

Testing pluripotency in vitro. By culturing aggregates of hESCs in suspension without bFGF for $3 \mathrm{wk}$, embryoid bodies (EBs) were formed. The presence of tissue originating from the three embryonic germ layers was demonstrated using RT-PCR and immunohistochemistry for marker genes of the three embryonic germ layers.

Immunostaining. Immunocytochemical characterization of new hESC lines was carried out using primary antibodies specific for Oct-4 (Chemicon), Nanog, TRA-1-60, TRA-181, SSEA-1, and SSEA-4 (all from Santa Cruz Biotechnology Inc., Santa Cruz, CA), as negative control human foreskin fibroblasts were used. Immunostaining was performed by fixating cells with 4\% paraformaldehyde in PBS for $20 \mathrm{~min}$ at room temperature and washed with PBS three times followed by blocking with 5\% FBS for $30 \mathrm{~min}$. Permeabilization was carried out using $5 \%$ blocking buffer consisting of $0.02 \%$ Triton X-100 (Sigma-Aldrich) in PBS. Primary antibodies were added to blocking buffer over night at $4^{\circ} \mathrm{C}$. To remove any unbound antibodies, the samples were washed three times with PBS. The secondary antibody, fluorescein isothiocyanate (FITC)-conjugated goat antimouse immunoglobulin $\mathrm{G}$ ( $\mathrm{IgG}$ ), Cy3-conjugated goat antirabbit $\mathrm{IgG}$, or FITC-conjugated bovine antigoat IgG (all from Chemicon) were diluted 1:200 in 5\% blocking buffer and added to cells for $60 \mathrm{~min}$ at room temperature in dark. After three washes with PBS, the cell nuclei were counterstained with Hoechst B2261 (SigmaAldrich) for $10 \mathrm{~min}$.

In addition to negative control cells, we used primary antibodies as a second negative control. Stained cells were analyzed using an Olympus IX71 inverted microscope
(Olympus Sverige AB, Solna, Sweden), and images were acquired with Olympus DP71 camera and the Soft Imagine Cell F version 2.6 Software (Olympus Sverige AB).

Staining of EBs was performed in a similar way after fixation with $4 \%$ paraformaldehyde for $1 \mathrm{~h}$. After fixation of the EBs, they were stained for markers specific for the three germ layers; bone morphogenetic protein-4 (BMP-4) for mesoderm (Novocasta Laboratories Ltd, Newcastle upon Tyne, UK), nestin (Chemicon) for ectoderm, and alpha-fetoprotein (AFP; Sigma-Aldrich) for endoderm. Primary antibodies were added in $5 \%$ blocking buffer over night at $4^{\circ} \mathrm{C}$ and later washed three times with PBS to remove any unbound antibodies. FITC-conjugated goat antimouse $\mathrm{IgG}$, Cy3-conjugated goat antirabbit $\mathrm{IgG}$, or FITC-conjugated bovine antigoat IgG (all from Chemicon) were used as secondary antibodies and were diluted (1:200) in $5 \%$ blocking buffer and applied to cells for $60 \mathrm{~min}$ at room temperature in dark. After two washes with PBS, the EBs were counterstained with Hoechst $33342(2 \mu \mathrm{g} / \mathrm{ml}$, Sigma) or PI $(1 \mu \mathrm{g} / \mathrm{ml}$, Sigma-Aldrich) for $10 \mathrm{~min}$ for nuclear staining.

RT-PCR analysis. RT-PCR was performed for BMP-4, nestin, and AFP. From the EBs, total RNA was extracted using Qiagen RNeasy Mini Kit (Qiagen, Germantown, Md) protocol as previously described (Strom et al. 2007).

\section{Results}

The derived lines are presented in Table 1. Two lines were derived from Day 5 embryos, 22 from Day 6, five from Day 7, and one from Day 8 blastocysts. The derivation process is illustrated in Fig. 1 in which the derivation of the line HS475 is shown. The lines HS181-HS429 have been described earlier, but the lines HS475, HS480, HS481, HS491, and HS539 are presented for the first time in this article.

All the lines have been banked. Most of the lines have been fully characterized, but the line HS402 was frozen before karyotyping, and teratoma formation due to lack of
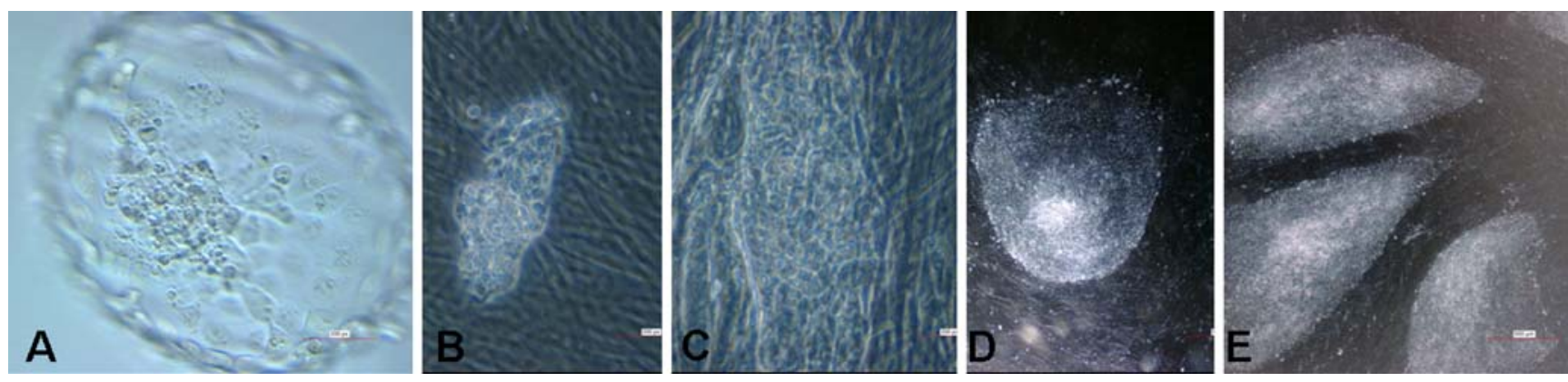

Figure 1. Derivation of the hESC line HS475. (A) Hatched blastocyst at Day 7 after fertilization, just before mechanical isolation of the ICM. (B) The ICM after derivation on human feeder layer. (C)
Outgrowth of the ICM, $5 \mathrm{~d}$ after derivation. $(D)$ HS475 at passage level 1. (E) HS475 at passage level 5. Magnification: $(A) 200 \mathrm{x}(B, C)$ $400 \mathrm{x}(D, E) 40 \mathrm{x}$ original magnification. 
Figure 2. Typical morphology of hESC colonies. (a) HS181 on Matrigel, $40 \mathrm{x}$ original magnification (b) HS401 on Matrigel, $400 \times(c)$ HS366 on hff, $40 \mathrm{x}$. (d) HS420 on hff, $400 \mathrm{x}$.
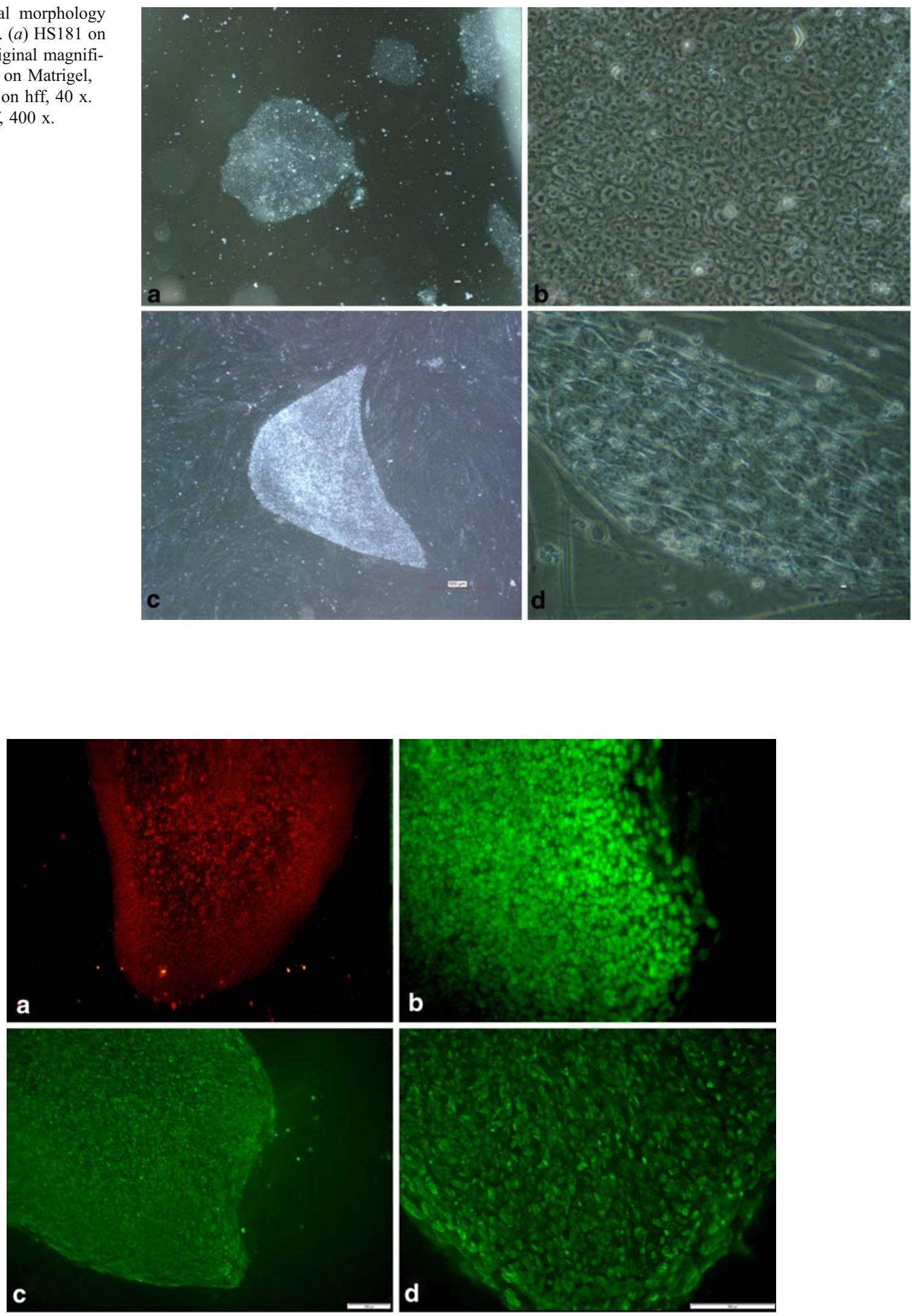

Figure 3. Characterization of hESC lines by immunocytochemistry. (a) HS401 p37 Positive staining for nuclear marker Nanog (b) 366 p35 staining for nuclear marker Oct 3/4 (c) 362 p36 staining for

TRA1-81 (d) 362 p36 staining for TRA-1-60. Magnification $(a, c)$ $100 \times(b, d) 200 \times$ original magnification. 
resources at the time of their derivation and the most recent HS475, HS480, HS481, HS491, and HS539 are in the process of these analyses. All these lines expressed all the studied pluripotency markers and markers of the three germ layers after in vitro differentiation to embryoid bodies. They are now in culture for karyotyping and teratoma formation. All the other lines expressed the pluripotency markers were pluripotent in both teratoma assay, and after in vitro differentiation to embryoid bodies. All the lines had a normal karyotype when for the first time analyzed. One of our lines, HS237, had a diisocentric X-chromosome at passage level as revealed by comparative genomic hybridization (Inzunza et al. 2004). In our hands, the other lines have remained karyotypically normal, but other teams have obtained abnormalities in the line HS181 (Inzunza et al. 2004) which has been the most widely distributed of our lines. The lines have been widely distributed into more than 100 laboratories. The lines HS181, HS207, HS237, HS293, HS306, HS346, HS415, HS420, HS422, HS426, and HS429 have been accepted by the European commission for use in the integrated project ESTOOLS. The most recent line, HS539, was obtained fresh from the preimplantation genetic diagnosis program of the IVF unit. The exclusion of Huntington's disease form the donated embryo was carried out indirectly using single nucleotide polymorphism assay because the parent did not want to know if she is a carrier of Huntington's disease. From the embryo donated for research, the exclusion could not be done.

Some of our oldest lines, among them HS181, have been kept timewise in culture for more than 200 passages, and the most resent one, HS539, has only been growing for seven passages.

Typical morphology of hESC colonies on hff and on Matrigel is shown in Fig. 2. Figure $2 a, b$ illustrates HS181 and HS401 in feeder-free culture on Matrigel and in mTeSR1 medium, and Fig. 2c, $d$ shows HS366 and HS420 on hff. Typical characterization by immunostaining from the lines HS362, HS366, and HS401 shows expression of Oct3/4, Nanog, TRA-1-60, and TRA-1-81 (Fig. 3).

\section{Discussion}

We have successfully derived a large number of lines with the aim of optimizing the quality of the hESC lines for various research projects and in preparation for clinical grade xeno-free good manufacturing practice (GMP) - quality hESC line derivation. Our present efforts are focused on feeder-free derivation, which would greatly simplify the GMP processes (Unger et al. 2008), but there still remain problems to be solved before safe clinical applications of hESC-differentiated cells are feasible. Tumorigenicity is the most important one (Blum and Benvenisty 2008).
It is important now to continue the work for derivation and culture of complete xeno-free hESC lines in fully defined conditions, not only for clinical aspects but also in order to obtain reliable results for basic research using the incomparable tool that the hESC lines are for us in a wide range of areas.

The great promise of the iPS cells does not make research on hESC redundant. Differentiation of iPS cells benefits from all protocols developed using hESC (Park et al. 2008). Tumorigenicity of iPS cell is still an open question.

Our present lines are not of clinical grade, but if culturing of old lines in GMP laboratories, to verify their safety, will be accepted by authorities, our lines offer a good selection of genotypes of lines which have been derived in cleaner conditions than most of the other reported lines. We can today offer a good number of hESC lines for the embryonic stem cell research community. More than 100 laboratories are using them. The lines are registered in the EU hESC registry (http://www.hescreg.eu/).

Open Access This article is distributed under the terms of the Creative Commons Attribution Noncommercial License which permits any noncommercial use, distribution, and reproduction in any medium, provided the original author(s) and source are credited.

\section{References}

Bjuresten K.; Hovatta O. Donation of embryos for stem cell research - how many couples consent? Hum. Reprod. 18(6): 1353-1355; 2003.

Blum B.; Benvenisty N. The tumorigenicity of human embryonic stem cells. Adv. Cancer Res. 100: 133-158; 2008.

Crook J. M.; Peura T. T.; Kravets L.; Bosman A. G.; Buzzard J. J.; Horne R.; Hentze H.; Dunn N. R.; Zweigerdt R.; Chua F.; Upshall A.; Colman A. The generation of six clinical-grade human embryonic stem cell lines. Cell Stem Cell 1(5): 490-494; 2007.

Fishel S. B.; Edwards R. G.; Evans C. J. Human chorionic gonadotropin secreted by preimplantation embryos cultured in vitro. Science 223(4638): 816-818; 1984.

Fridstrom M.; Carlstrom K.; Sjoblom P.; Hillensjo T. Effect of prednisolone on serum and follicular fluid androgen concentrations in women with polycystic ovary syndrome undergoing in-vitro fertilization. Hum. Reprod. 14(6): 1440-1444; 1999.

Hovatta O.; Mikkola M.; Gertow K.; Stromberg A. M.; Inzunza J.; Hreinsson J.; Rozell B.; Blennow E.; Andang M.; AhrlundRichter L. A culture system using human foreskin fibroblasts as feeder cells allows production of human embryonic stem cells. Hum. Reprod. 18(7): 1404-1409; 2003.

Inzunza J.; Gertow K.; Stromberg M. A.; Matilainen E.; Blennow E.; Skottman H.; Wolbank S.; Ahrlund-Richter L.; Hovatta O. Derivation of human embryonic stem cell lines in serum replacement medium using postnatal human fibroblasts as feeder cells. Stem Cells 23(4): 544-549; 2005.

Inzunza J.; Sahlen S.; Holmberg K.; Stromberg A. M.; Teerijoki H.; Blennow E.; Hovatta O.; Malmgren H. Comparative genomic hybridization and karyotyping of human embryonic stem cells 
reveals the occurrence of an isodicentric $\mathrm{X}$ chromosome after long-term cultivation. Mol. Hum. Reprod. 10(6): 461-466; 2004.

Koivisto H.; Hyvarinen M.; Stromberg A. M.; Inzunza J.; Matilainen E.; Mikkola M.; Hovatta O.; Teerijoki H. Cultures of human embryonic stem cells: serum replacement medium or serumcontaining media and the effect of basic fibroblast growth factor. Reprod. Biomed. Online 9(3): 330-337; 2004.

Martin M. J.; Muotri A.; Gage F.; Varki A. Human embryonic stem cells express an immunogenic nonhuman sialic acid. Nat. Med. 11(2): 228-232; 2005.

Martin-Ibanez R.; Unger C.; Stromberg A.; Baker D.; Canals J. M.; Hovatta O. Novel cryopreservation method for dissociated human embryonic stem cells in the presence of a ROCK inhibitor. Hum. Reprod. 23(12): 2744-2754; 2008.

Park I. H.; Arora N.; Huo H.; Maherali N.; Ahfeldt T.; Shimamura A.; Lensch M. W.; Cowan C.; Hochedlinger K.; Daley G. Q. Diseasespecific induced pluripotent stem cells. Cell 134(5): 877-886; 2008.

Reubinoff B. E.; Pera M. F.; Fong C. Y.; Trounson A.; Bongso A. Embryonic stem cell lines from human blastocysts: somatic differentiation in vitro. Nat. Biotechnol. 18(4): 399-404; 2000.
Reubinoff B. E.; Pera M. F.; Vajta G.; Trounson A. O. Effective cryopreservation of human embryonic stem cells by the open pulled straw vitrification method. Hum. Reprod. 16(10): 2187-2194; 2001.

Rodin, S., Domogatskaya, A., Ström S., Hansson E. M., Chien K. R., Inzunza, J., Hovatta, O., Tryggvason, K. Long-term selfrenewal of human embryonic stem cells on human recombinant laminin-511 in xeno-free and feeder-free environment. Nat Biotechnol.; 2010 (accepted).

Solter D.; Knowles B. B. Immunosurgery of mouse blastocyst. Proc. Natl. Acad. Sci. USA 72(12): 5099-5102; 1975.

Strom S.; Inzunza J.; Grinnemo K. H.; Holmberg K.; Matilainen E.; Stromberg A. M.; Blennow E.; Hovatta O. Mechanical isolation of the inner cell mass is effective in derivation of new human embryonic stem cell lines. Hum. Reprod. 22(12): 3051-3058; 2007.

Thomson J. A.; Itskovitz-Eldor J.; Shapiro S. S.; Waknitz M. A.; Swiergiel J. J.; Marshall V. S.; Jones J. M. Embryonic stem cell lines derived from human blastocysts. Science 282(5391): 1145-1147; 1998.

Unger C.; Skottman H.; Blomberg P.; Dilber M. S.; Hovatta O. Good manufacturing practice and clinical-grade human embryonic stem cell lines. Hum. Mol. Genet. 17(R1): R48-R53; 2008. 